\title{
A novel mechanism regulating growth factor association with the cell surface: identification of a PDGF retention domain
}

\author{
William J. LaRochelle, Mary May-Siroff, Keith C. Robbins, ${ }^{1}$ and Stuart A. Aaronson \\ Laboratory of Cellular and Molecular Biology, National Cancer Institute and the ${ }^{1}$ Laboratory of Cellular Development and \\ Oncology, National Institute of Dental Research, National Institutes of Health, Bethesda, Maryland 20892 USA
}

\begin{abstract}
Platelet-derived growth factor (PDGF) chimeras were used to map a domain responsible for either efficient secretion of PDGF-A or the tight cell association of PDGF-B to their carboxy-terminal domains. Introduction of stop codons within PDGF-A or PDGF-B further dissected their respective carboxy-terminal domains. Although successive deletions of the PDGF-A carboxyl terminus did not impair its secretion, incremental deletions from the carboxyl terminus of PDGF-B abrogated its membrane retention properties and promoted secretion. By this approach, PDGF-B retention properties could be localized to PDGF-B residues 212-226. A processed form of PDGF-B, which contained this domain, was expressed at the cell surface but not released. Comparison of PDGF-B with PDGF-A revealed an analogous sequence located at the PDGF-A carboxyl terminus. We demonstrated that this PDGF-A domain also acts as a retention sequence under conditions that inhibit its proteolytic cleavage. Thus, differences in PDGF-A and PDGF-B secretion relate to differential proteolytic processing of analogous retention domains. All of these findings establish a new mechanism for stable growth factor presentation at the cell surface.
\end{abstract}

[Key Words: Platelet-derived growth factor; development; cell surface association; retention sequence; protein structure]

Received January 25, 1991; revised version accepted April 17, 1991.

The ability of cells to communicate with one another is of critical importance in development and differentiation. Among the various mechanisms described for intercellular communication, the interactions of growth factors and their cell-surface receptors are fundamentally indispensable. Accumulating evidence indicates that certain transmembrane glycoproteins are cleaved to yield soluble growth factors. For example, members of the epidermal growth factor (EGF) family are synthesized as transmembrane proteins with variable numbers of EGF repeats in their extracellular domains (for review, see Massague 1990). The Drosophila Notch (Gray et al. 1983; Scott et al. 1983), Delta, and crumbs gene products, as well as the nematode Caenorhabditis elegans lin-12 and glp-1 gene products, encode membraneanchored proteins with multiple EGF-like repeats but are not known to be cleaved as soluble factors (Massague 1990). Other growth factors, including colony-stimulating factor (CSF)-1 (Rettenmier and Roussel 1988) and the c-kit ligand (Flanagan and Leder 1990; Huang et al. 1990), are encoded by alternative transcripts yielding either transmembrane or secreted forms. Presumably, this allows for growth factor action proximate to the producing cell or at a distance. In fact, recent evidence indicates that a genetic lesion that specifically deletes expression of the cell surface form of the c-kit ligand is associated with certain developmental abnormalities in the mouse (Flanagan et al. 1991).

One of the earliest examples of a growth factor that retains a tight cell association was the $\mathrm{v}$-sis gene product (Robbins et al. 1985), whose human homolog is plateletderived growth factor (PDGF)-B chain (Devare et al. 1983; Doolittle et al. 1983; Waterfield et al. 1983). Like the transmembrane growth factor precursors, the v-sis gene product traverses the normal secretory pathway and achieves a cell surface location. However, the v-sis gene product/PDGF-B lacks a transmembrane sequence that could readily account for its cell retention. Later studies revealed that the structurally related growth factor PDGF-A, whose primary translational product also lacks a transmembrane domain, is actively secreted by the cell (Beckman et al. 1988). Molecular genetic studies have localized PDGF-A and PDGF-B regions that confer their different secretion/retention properties to their carboxyterminal regions (LaRochelle et al. 1990). The present studies were undertaken in an effort to elucidate the molecular mechanism that accounts for these very different secretory properties of PDGF-A and PDGF-B. Our find- 
ings define a novel mechanism by which growth factor expression can be targeted for action either near or at a distance from the synthesizing cell.

\section{Results}

PDGF chimeras localize the domain responsible for differences in the secretory properties of PDGF-A and PDGF-B homodimers

Biosynthesis, assembly, and processing of the v-sis gene product and c-sis/PDGF-B are very similar (Robbins et al. 1985; Beckman et al. 1988). Each is translated as a precursor protein that is first detected in the endoplasmic reticulum and Golgi apparatus. Cleavage of both proteins occurs sequentially at their amino and then carboxyl termini. In the case of the v-sis gene product, proteolytic cleavage has been shown to occur specifically at the cell surface (Robbins et al. 1985). Both the v-sis gene product and human PDGF-B are retained by the cell and not released into culture fluids (Robbins et al. 1985; Beckman et al. 1988; LaRochelle et al. 1990). PDGF-A is known to be processed at its amino terminus and is efficiently secreted from expressing cells (Beckman et al. 1988).

We have shown previously that differences in secretory properties map to the carboxy-terminal domains of PDGF-A and PDGF-B. Translational products from PDGF chimeras containing PDGF-A codons 178-211 were efficiently released from the cell, whereas those containing PDGF-B codons 178-241 were retained in association with the cell membrane fraction /LaRochelle et al. 1990). Thus, the carboxyl terminus of PDGF-A may promote secretion or the analogous domain of PDGF-B may be responsible for cellular retention. In an effort to map precisely this property of the PDGFs, we engineered PDGF chimeras that switched the carboxy-terminal regions of PDGF-A and PDGF-B within the proposed secretion/retention domain.

As a measure of biological function, we analyzed the transforming properties of parental and recombinant PDGFs by expression in NIH-3T3 cells under the control of the metallothionein promoter. The mouse metallothionein vector, MMTneo, also contained a dominant selectable neomycin marker gene, making it possible to score neomycin-resistant colony formation as well. Thus, we were able to determine precisely the specific transforming activity for each construct, as well as select mass populations for analysis of PDGF translational products.

Each parental and chimeric molecule demonstrated biological activity in the transformation assay (Fig. 1). PDGF-B had an $\sim 25$-fold higher transforming efficiency than that of PDGF-A. As expected, PDGF- $\mathrm{B}^{188} \mathrm{~A}^{187}$, which contained the minimal transforming domain of PDGF-B, was potently transforming like PDGF-B, while PDGF- $\mathrm{A}^{187} \mathrm{~B}^{190}$, containing the analogous domain of PDGF-A, was weakly transforming like PDGF-A.

To compare the compartmentalization of PDGF$\mathrm{A}^{187} \mathrm{~B}^{190}$ and PDGF- ${ }^{188} \mathrm{~A}^{187}$, mass-selected populations of each transfectant were metabolically labeled for $4 \mathrm{hr}$, and the crude membrane fractions were subjected to immunoprecipitation analysis using antisera specific to the PDGF-A or PDGF-B amino- or carboxy-terminal regions, respectively. As shown in Figure 1, the primary PDGF-A translational product $\mathrm{p} 42$, as well as its $38-$ and $32-\mathrm{kD}$ processed forms, were detected in the membrane fraction of PDGF-A-transfected cells. Similarly, the primary PDGF-B translational product p54 was processed at amino and carboxyl termini, respectively, to 34 - and 24$\mathrm{kD}$ species, as reported previously (Beckman et al. 1988; LaRochelle et al. 1990). The PDGF-A ${ }^{187} \mathrm{~B}^{190}$ chimera encoded a p45 primary translational product and was processed at amino and carboxyl termini to 40-, 36-, and 26-kD species (Fig. 1). The reciprocal chimeric construct $\mathrm{B}^{188} \mathrm{~A}^{187}, \mathrm{p} 43$, was amino-terminally processed to a $\mathrm{p} 30$ species. Thus, each transfectant expressed proteins having the expected PDGF-A or PDGF-B antigenic determinants, as well as predicted sizes and processing relative to PDGF-A or PDGF-B.

To compare their secretion/retention properties, transfectants containing the chimeras were metabolically labeled and conditioned medium was immunoprecipitated with the same series of antibodies. Figure 1 shows that PDGF-A and PDGF- $\mathrm{B}^{188} \mathrm{~A}^{187}$ were actively secreted, as major p32 and p30 species, respectively, whereas PDGF$\mathrm{B}$ and the reciprocal PDGF- $\mathrm{A}^{187} \mathrm{~B}^{190}$ were $>90 \%$ retained. These findings localized the secretion/retention domain as distal to amino acid 187 of PDGF-A or amino acid 190 of PDGF-B.

\section{Deletions of the PDGF-A carboxy-terminal domain do not affect its secretory properties}

To investigate the role of the PDGF-A carboxyl terminus in secretion, we generated three PDGF-A mutants in which stop codons were introduced at or beyond codon 187. Transfection analysis showed that introduction of stop codons and successive deletion of the carboxy-terminal coding sequence had no effect on the transforming activity of any of these mutants. Each possessed a low transforming efficiency comparable to that of wild-type PDGF-A (Fig. 2). PDGF-A lacking codons $1-86$ has been reported to retain biologic activity (Ostman et al. 1988). Because PDGF-A stop 186 retained focus-forming activity (Fig. 2), the transforming domain of PDGF-A (amino acid residues 86-186) appears to be analogous to that reported for PDGF-B (King et al. 1985).

Marker-selected cultures of PDGF-A and PDGF-A stop 204, 196, and 186 were next analyzed for the compartmentalization of PDGF immunoreactive proteins. As shown in Figure 2, cell membrane fractions of each transfectant contained the expected protein species. PDGF-A was detected as a predominant $\mathrm{p} 42$ form, while PDGF-A stop 204, 196, and 186 were synthesized as p40, p39, and p37 species, respectively, due to progessive deletion of carboxy-terminal sequence (Fig. 2). The wildtype PDGF-A gene product was detected in the conditioned medium as an efficiently secreted amino-terminally processed p32 species, while PDGF-A stop 204, 


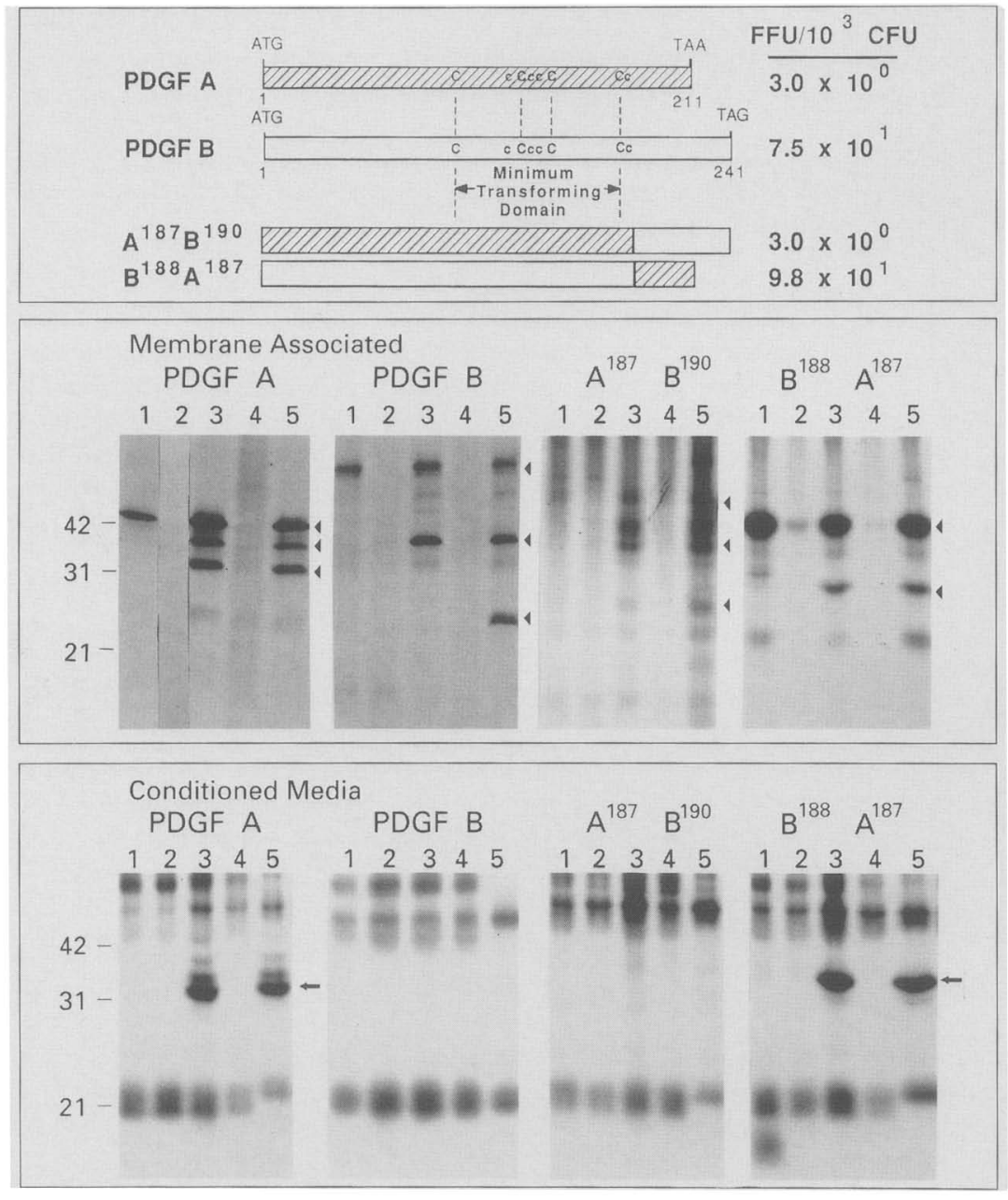

Figure 1. Transfection and expression of PDGF chimeras. Chimeric PDGF molecules (top) were constructed by recombination of the PDGF-A (hatched box) and PDGF-B (open box) genes at a common preexisting or engineered restriction endonuclease site as described in Materials and methods. Transformation efficiency for DNA constructs is shown as the number of focus-forming units (FFU) divided by colony-forming units (CFU). Transfected cultures were selected for their ability to grow in G418 and were labeled metabolically. Crude membranes (middle) or conditioned media (bottom) were immunoprecipitated by using antisera directed against PDGF-A residues 7287 (amino-terminal) or 185-199 (carboxy-terminal), PDGF-B residues 23-37 (amino-terminal) or 227-241 (carboxy-terminal), or purified PDGF (lane 5). Use of amino (lanes 1 and 2)- or carboxy (lanes 3 and 4)-terminal antisera to PDGF-A or PDGF-B was determined by the composition of the transfected gene. In some cases (lanes 2 and 4), antibodies were preincubated with excess homologous peptide. Immune complexes were analyzed by SDS-PAGE under nonreducing conditions, and results were visualized by autoradiography.
196, and 186 transfectants demonstrated readily detectable PDGF-A immunoreactive species of p30, p28, and p26, respectively (Fig. 2). As with PDGF-A, each of the stop mutants was amino-terminally processed. These results demonstrated that the progressive deletions of the PDGF-A carboxyl terminus had no effect on its processing or secretory properties.

Transfectants expressing PDGF-A stop 204, 196, and 186 were also analyzed for biologically active PDGF in crude membrane fractions and culture fluids. In each case, mitogenic activity from NIH-3T3 cells was readily detectable in culture fluids at levels comparable to that of the PDGF-A transfectant (data not shown). Specific inhibition of the mitogenic activity by a PDGF-neutralizing antibody in each case confirmed the PDGF-related nature of the growth factor. All of these findings excluded the possibility that the carboxy-terminal domain of PDGF-A was required to promote its active secretion.

\section{Mapping of a PDGF-B subdomain responsible for its membrane retention}

To investigate the role of the PDGF-B carboxyl terminus in secretion, we generated four sequential stop mutants at or beyond PDGF-B codon 185 (Fig. 3). When the biologic activities of these mutants were analyzed, each demonstrated potent transforming activity comparable with the parental PDGF-B (Fig. 3).

Analysis of PDGF immunoreactive proteins in crude membrane fractions revealed expression of each mutant protein at levels comparable to that of the wild-type PDGF-B protein. Moreover, the size of each mutant was consistent with the predicted truncation. Thus, PDGF-B was observed as a predominant $54-\mathrm{kD}$ species, while PDGF-B stop $235,227,211$, and 185 gene products migrated as p53 (data not shown), p51, p48, and p45 species, respectively (Fig. 3). When we analyzed culture fluids of the same transfectants for PDGF-B immunoreactive proteins, striking differences were observed, as shown in Figure 3. Whereas wild-type PDGF-B, stop mutant 235, or stop mutant 227 was not detectably released, aminoterminally processed p31 and p27 products of PDGF-B stop 211 and 185 mutants, respectively, were readily observed. These findings strongly suggested that PDGF-B amino acid residues $212-226$ were responsible for the tight membrane association of the PDGF-B molecule.

To confirm the secretory properties assigned to each mutant protein, we measured the release of biologically 


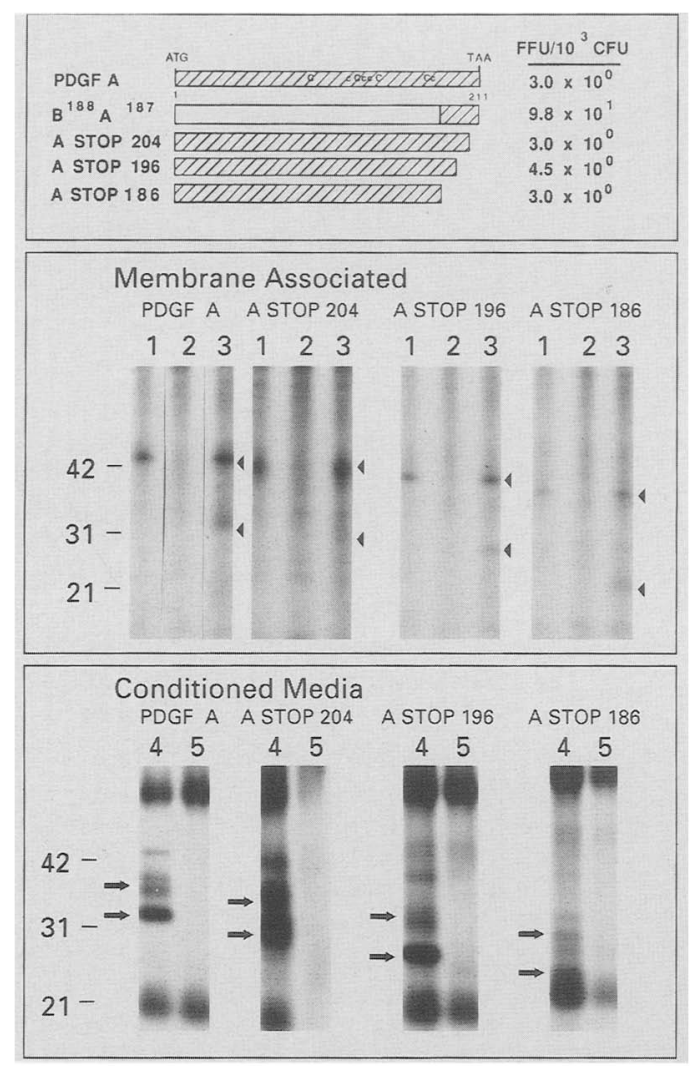

Figure 2. Transfection and expression of PDGF-A stop mutants. PDGF-A stop mutants were constructed by introduction of a translocational termination signal at the designated codon (top). Transformation efficiency was assayed as described in Materials and methods. Transfected cultures were selected for their ability to grow in G418 and were labeled metabolically. Crude membrane fractions (middle) or conditioned media (bottom) were immunoprecipitated with PDGF-A amino-terminal antiserum to residues 72-87 (lanes 1 and 2), PDGF antibody (lanes 3 and 4), or control goat antibody (lane 5). In some cases (lane 2), antibodies were preincubated with excess amino-terminal peptide. Major processed forms of PDGF-A are identified by arrows. Minor partially processed higher molecular weight forms were also observed. Immune complexes were analyzed by SDS-PAGE under nonreducing conditions, and results were visualized by autoradiography.

active growth factor into conditioned media. As a control for these studies, the level of PDGF mitogenic activity associated with crude membrane fractions was examined as well. As shown in Figure 4, mitogenically active PDGF-B was recovered in the crude membrane fractions from each of the PDGF-B stop mutants. Whereas no detectable mitogenic activity was detectable in medium from PDGF-B or PDGF-B stop mutants 235 or 227 , mitogenic activity was readily detectable in conditioned medium from PDGF-B stop mutant 211 or 185 . The complete inhibition of mitogenic activity in each case by PDGF-neutralizing antibody confirmed the identity of the growth factor as PDGF. All of these findings were consistent with our immunoprecipitation analysis and identified PDGF-B amino acid residues $212-226$ as responsible for its membrane retention.

PDGF-B electrostatically interacts with cell membranes and exhibits biochemical properties of a peripheral membrane protein

To investigate directly the biochemical nature of PDGF$B$ interaction with cell membranes, crude membrane fractions were subjected to biochemical treatments known to remove peripheral membrane proteins (Table 1). PDGF-B remained membrane associated under conditions of low ionic strength or by conditions that remove divalent cations such as treatment with EDTA. However, at $0.25 \mathrm{M} \mathrm{NaCl}$, a portion of PDGF-B could be dis-

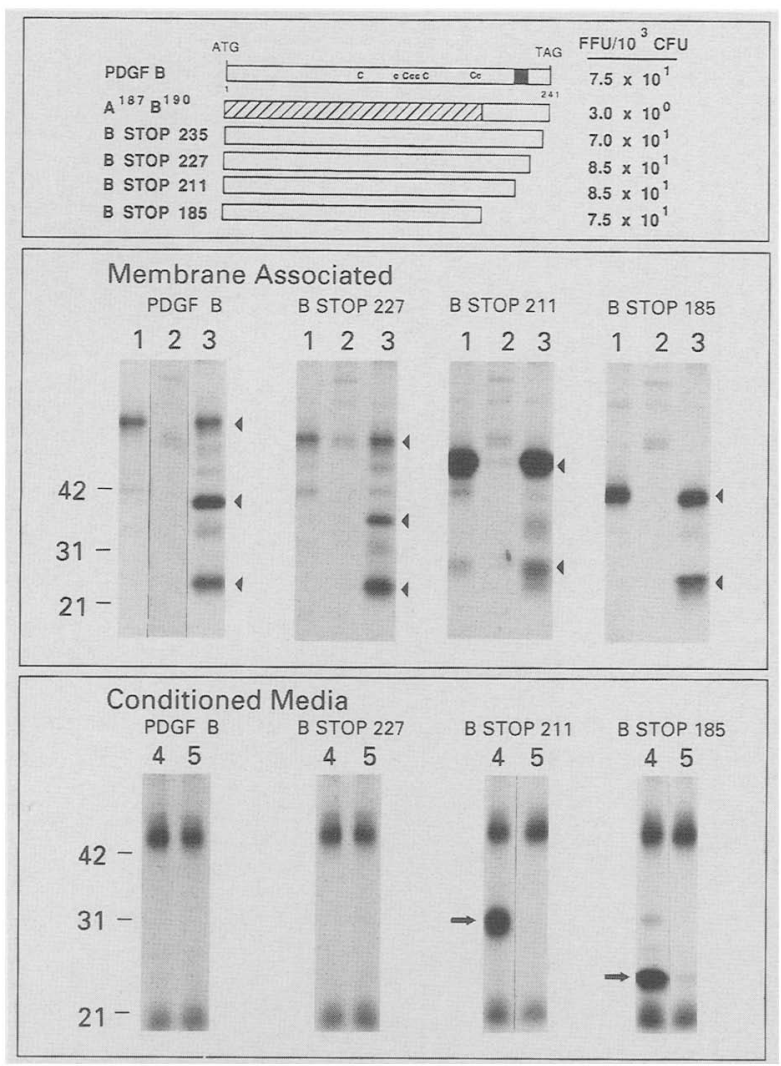

Figure 3. Transfection and expression of PDGF-B stop mutants. PDGF-B stop mutants were constructed by introduction of a translocational termination signal at the designated codon (top). Transformation efficiency was assayed as described in Materials and methods. Transfected cultures were selected for their ability to grow in G418 and were labeled metabolically. Crude membrane fractions (middle) or conditioned medium (bottom) were immunoprecipitated with PDGF-B amino-terminal antiserum to residues 23-37 (lanes 1 and 2), PDGF antibody (lanes 3 and 4), or control goat antibody (lane 5). As an additional control, antibodies were preincubated with excess amino-terminal peptide (lane 2). Major processed forms of PDGF-B are identified by arrows. Immune complexes were analyzed by SDSPAGE under nonreducing conditions, and results were visualized by autoradiography. 


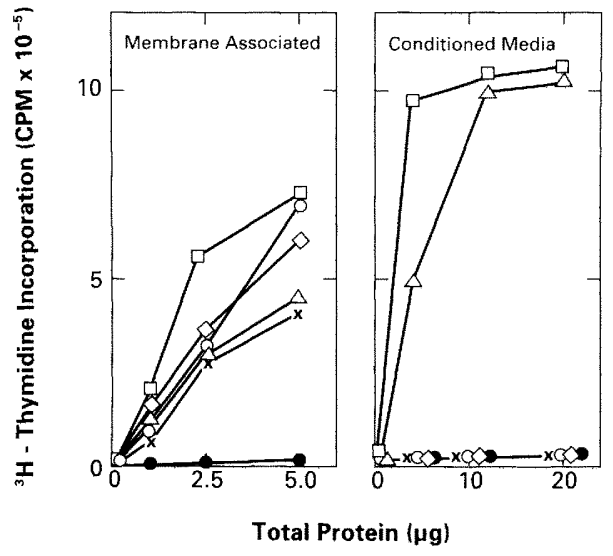

Figure 4. Mitogenic activity of PDGF-B stop mutant proteins from crude membrane fractions or conditioned media. Quiescent NIH-3T3 cells were exposed to crude membrane fractions (left) or conditioned medium (right) from PDGF-B (O), B stop $235(\times)$, B stop $227(\diamond)$, B stop $211(\square)$, or B stop $186(\Delta)$ transfectants or NIH-3T3 cells (O). Cultures were examined $18 \mathrm{hr}$ later for their ability to incorporate $\left[{ }^{3} \mathrm{H}\right]$ thymidine, as described in Materials and methods.

lodged from the membrane. Increasing salt concentration above $0.5 \mathrm{M}$ removed $>90 \%$ of the molecule. Suramin, a highly charged anionic molecule, readily released $>95 \%$ of PDGF-B from membranes. Other conditions known to remove peripheral membrane proteins, such as $0.1 \mathrm{~N} \mathrm{NaOH}$, also stripped PDGF-B from the cell membrane fraction. All of these results support the conclusion that PDGF-B interacts with cell membranes in an electrostatic rather than a hydrophobic manner.

\section{Its retention sequence localizes PDGF-B} to the cell surface

We utilized two independent approaches in an effort to localize the site of PDGF-B retention by the cell. Metabolically labeled PDGF-B transfectants were either lysed

Table 1. Removal of sis proteins from cell membranes

\begin{tabular}{lc}
\hline Treatment & Percent removal \\
\hline $10 \mathrm{mM}$ sodium phosphate $(\mathrm{pH} 7.4)$ & $<2$ \\
$10 \mathrm{~mm}$ EDTA $(\mathrm{pH} 7.4)$ & $<2$ \\
$0.25 \mathrm{M} \mathrm{NaCl}$ & $20-40$ \\
$0.50 \mathrm{M} \mathrm{NaCl}$ & $>90$ \\
$1.0 \mathrm{M} \mathrm{NaCl}$ & $>90$ \\
$0.1 \mathrm{~N} \mathrm{NaOH}$ & $>90$ \\
$100 \mu \mathrm{M} \mathrm{Suramin}$ & $>90$ \\
$1.0 \% \mathrm{SDS}$ & $>90$
\end{tabular}

A radiolabeled crude cell membrane fraction was treated as indicated in Materials and methods and fractionated, and the amount of radiolabeled proteins in the membrane pellet was compared to that in the membrane supernatant by immunoprecipitation analysis. prior to or following exposure to PDGF antibody at $4^{\circ} \mathrm{C}$ and extensive washing. As shown in Figure 5, cell lysates contained major PDGF-B immunoreactive species of 54 , 34 , and $24 \mathrm{kD}$ under nonreducing conditions. In contrast, PDGF antibody absorption to the living cells led to the specific detection of the $34-\mathrm{kD}$ species. These results suggested that the processed $34-\mathrm{kD}$ form of PDGF-B was located at the cell surface, whereas the unprocessed 54$\mathrm{kD}$ and processed $24-\mathrm{kD}$ forms were antibody inaccessible, presumably because of their location within the cell.

To confirm these findings, PDGF-B-transfected NIH3T3 cells were labeled in the presence or absence of suramin, which was efficient in releasing PDGF-B from crude cell membranes (Table 1). Whereas PDGF-B was detectable in conditioned medium from cells radiolabeled in the absence of suramin (Fig. 5), suramin treatment caused specific release of the $34-\mathrm{kD}$ species. Consistent with the results of antibody studies, neither the p54 nor p24 species was detectable under these conditions. Because p34 was specifically detected by the PDGF-B carboxy-terminal antiserum, these findings demonstrate that $\mathrm{p} 34$, which contains the PDGF-B retention sequence identified by us, must achieve a cell surface location. Moreover, the release of the molecule by suramin further substantiates the concept that its retention sequence is electrostatic in nature.

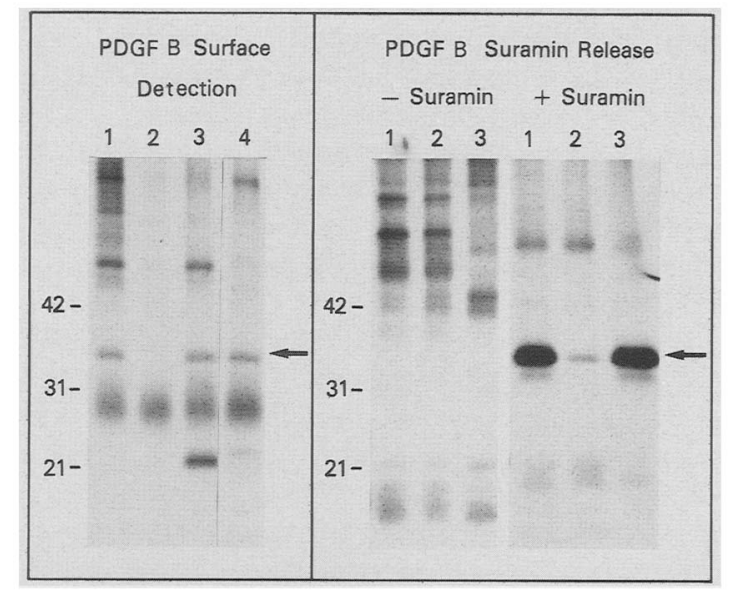

Figure 5. Cell-surface antibody absorption and suramin release of PDGF-B. NIH-3T3 cells transfected with the PDGF-B gene were radiolabeled, lysed, and immunoprecipitated with PDGF-B carboxy-terminal antiserum to residues 227-241 (lanes 1 and 2), or PDGF antibody (lane 3). An equivalent number of radiolabeled cells was incubated with PDGF antibody, washed free of unbound antibody, lysed, and immunoprecipitated (left; lane 4). For suramin extraction experiments (right), whole cells were radiolabeled in the absence or presence of suramin. PDGF-B released by suramin treatment was determined by immunoprecipitation of conditioned media with carboxy-terminal antiserum (lanes 1 and 2) or PDGF antibody (lane 3). As an additional control, antiserum was preincubated with excess carboxy-terminal peptide (lane 2). Immune complexes were analyzed by SDS-PAGE under nonreducing conditions, and results were visualized by autoradiography. 
Differential proteolytic processing of retention sequences within PDGF-A and PDGF-B explain their secretory properties

The sequence of PDGF-A from amino acid residues 192206 is similar to that of the PDGF-B retention sequence, and potential sites for proteolytic cleavage reside within both the PDGF-B retention sequence and the analogous PDGF-A domain (Fig. 6, top). We reasoned that differences in the secretory properties of PDGF-A and PDGF-B might be explained either by the specific amino acids that define the PDGF-B retention sequence or by differences in proteolytic processing of analogous functional domains within both molecules. To investigate these possibilities, we exposed PDGF-A transfectants to monensin, which has been shown to inhibit proteolytic processing of the v-sis/PDGF-B gene product at the cell surface (Robbins et al. 1985). Under these conditions (Fig. 6, middle), only the nonprocessed $\mathrm{p} 42$, species was detected in membrane fractions immunoprecipitated either with PDGF-A antisera directed against residues $185-199$ or the carboxy-terminal 12 residues $(200-211)$, which contained residues analogous to the PDGF-B retention sequence (Fig. 6 , top). These findings were in striking contrast to results in untreated cells, in which processed p 38 and p32 species, as well as p42, were readily observed in membrane fractions (Fig. 1). Thus, monensin efficiently blocked PDGF-A proteolytic processing. These same antisera, as well as our broadly reactive PDGF antibody, detected little if any PDGF-A release into conditioned medium of monensin-treated cells, in marked contrast with the efficient release of PDGF-A by untreated cultures (Fig. 1). Therefore, monensin exposure also efficiently inhibited PDGF-A secretion. All of these results demonstrated that nonprocessed PDGF-A was retained in membranes of monensin-treated cells and that the retained protein was not proteolytically processed at its carboxyl terminus.

To determine whether nonprocessed PDGF-A p42 remained cell associated in a manner analogous to that of PDGF-B, we extracted p42 by exposure of monensintreated membranes to suramin. As shown in Figure 6 (bottom left), nonprocessed p42 was readily released in response to suramin. Moreover, the protein released by suramin treatment was recognized by antisera to 185 199 or 200-211. Thus, nonprocessed PDGF-A p42, which contained the carboxy-terminal domain analogous to the PDGF-B retention sequence, was retained in the membrane in a manner indistinguishable from that of PDGF-B.

To further analyze the mechanistic basis for efficient PDGF-A secretion under normal conditions, we tested the translational products released from untreated PDGF-A transfectants for recognition by our panel of antibodies. As shown in Figure 6 (bottom right), antiserum to residues $185-199$, as well as antibody to PDGF, readily detected three secreted forms of PDGF-A, including $\mathrm{p} 41, \mathrm{p} 38$, and $\mathrm{p} 32$, whereas antiserum to carboxyterminal residues $200-211$ recognized none of these secreted forms. These findings strongly argue that prote-

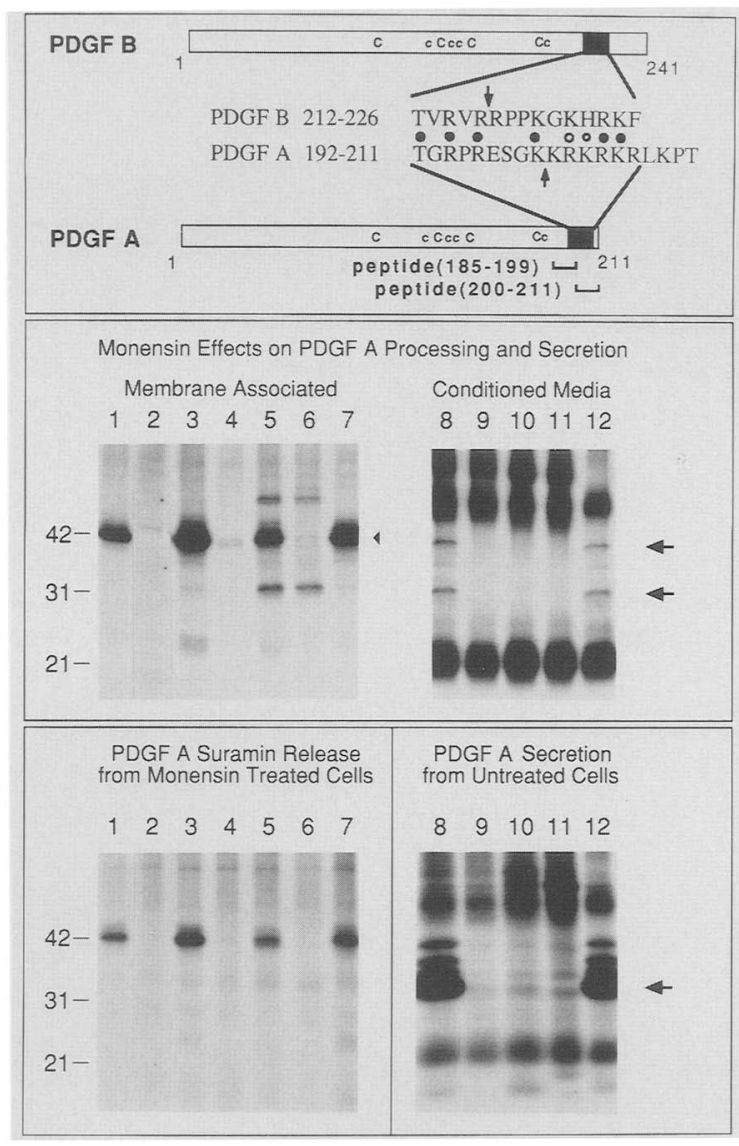

Figure 6. Monensin effects on PDGF-A processing and secretion. (Top) The PDGF-B retention sequence is shown with the analogous sequence of PDGF-A. (O) Identical amino acid residues in these domains; $|O|$ conservative substitutions. The locations of PDGF-A residues 185-199 or 200-211 are also shown. Cysteine residues within the minimal transforming domain designated essential $(C)$, or nonessential (c) for PDGF-B transformation are illustrated. A potential proteolytic cleavage site for each PDGF chain is designated by an arrow. (Middle) PDGFA transfectants were cultured with monensin and metabolically labeled as described in Materials and methods. Crude membrane fractions or conditioned media were analyzed by immunoprecipitation. (Bottom) For suramin release of cell membrane-associated PDGF-A (bottom left), crude cell membranes were prepared from monensin-treated cells that were labeled metabolically as described in Materials and methods. Protein was extracted from membranes with suramin, and the released PDGF-A was immunoprecipitated. Conditioned medium from untreated cells (bottom right) was also immunoprecipitated. Antisera to PDGF-A peptide 72-87 (lanes 1 and 2), peptide 185199 (lanes 3, 4, 8 and 9), peptide 200-211 (lanes 5, 6, 10, and 11), or PDGF antibody (lanes 7 and 12) were utilized. In some cases (lanes $2,4,6,9$, and 11), antisera were incubated with an excess of homologous peptide. Immune complexes were analyzed by SDS-PAGE under nonreducing conditions, and results were visualized by autoradiography for 3 days.

olytic processing of the extreme PDGF-A carboxyl terminus involving residues $200-211$ is required for efficient PDGF-A secretion and that differences in PDGF- 
A and PDGF-B secretion by fibroblasts relate to differences in proteolytic processing of analogous retention domains.

\section{Discussion}

There is increasing evidence that growth factors exist in forms that are either efficiently secreted or tightly retained in close association with the cell. Presumably, such variants allow for biologic action either locally or at a distance during development, tissue remodeling, and repair. To date, understanding the basis for growth factor retention and presentation at the cell surface has been limited to those growth factors that possess transmembrane domains. Included in this category are members of the EGF family (Massague 1990), as well as CSF-1 (Rettenmier and Roussel 1988 ) and c-kit ligands (Huang et al. 1990|. As structures of additional growth factors have been elucidated, it has been increasingly evident that some factors that lack transmembrane sequences can exhibit tight cell association. Our present findings establish a novel mechanism for retention at the cell surface of such growth factors. A stretch of positively charged amino acid residues at the PDGF carboxyl terminus retains this growth factor for action on the same or adjacent cells or possibly as a migratory anchor for cells expressing the appropriate receptors.

Several lines of evidence made it possible to map the PDGF retention sequence to a small stretch of positively charged amino acids. In the case of PDGF-B, deletion of this domain or its substitution by the analogous domain of PDGF-A led to efficient growth factor release from the cell. Conversely, retention properties were conferred to the PDGF-A molecule by substitution with regions of the PDGF-B molecule containing this domain. Finally, we demonstrated that a processed form of PDGF-B, which retained this domain, becomes cell surface associated but is not released. All of these findings argue that PDGF-B is exposed at the cell surface but is retained by virtue of this small stretch of positively charged amino acids.

A sequence structurally similar to that of the PDGF-B retention sequence was identified at the carboxyl terminus of PDGF-A (Fig. 6). We were able to establish that this PDGF-A domain acts in a manner analogous to that of the PDGF-B retention sequence to retain PDGF-A at a cell membrane location in the absence of proteolytic processing. Thus, inhibition of PDGF-A proteolytic processing was associated with its lack of release into culture fluids, whereas normal processing of the molecule led to efficient release. Furthermore, we found that the PDGFA species secreted were not recognized by antisera against this retention domain but were detected by antisera to an immediately adjacent upstream domain. Thus, immunochemical analysis made it possible to map the processing site within or immediately upstream of this carboxy-terminal sequence. All of these findings establish that secretion of PDGF-A and PDGF-B is regulated differentially at the level of cell-surface proteolysis of functionally analogous retention domains.
There is an endothelial form of PDGF-A, which represents an alternatively spliced transcript (Rorsman et al. 1988). The transcript for this alternative form specifically lacks the exon that contains the PDGF-A retention sequence. Previous studies have shown that this form of PDGF-A is secreted efficiently (Beckman et al. 1988). Thus, the endothelial form of PDGF-A would have the capacity to be efficiently secreted even from cells that lack the ability to process the alternative PDGF-A containing the retention domain.

The retention sequence identified in this study differs from previously identified sequences that target certain molecules for retention within the endoplasmic reticulum (ER) or Golgi. For example, the consensus KDEL sequence has been implicated in the retention of soluble resident proteins of the ER (Munro and Pelham 1987). ER resident transmembrane proteins appear to be retained by a positively charged stretch of amino acids at their carboxyl terminus (Nilsson et al. 1989; Jackson et al. 1990). However, the targeting sequences of such transmembrane proteins reside in their cytoplasmic portions and are thought to act as signals for intracellular machinery responsible for their sequestration in the ER (Nilsson et al. 1989; Jackson et al. 1990). In contrast, the entire PDGF-B is translocated into the lumen of the ER and, thus, would not be accessible to such a cytoplasmic retention mechanism.

Highly basic amino acid stretches have also been implicated in nuclear targeting of a number of molecules (Kalderon et al. 1984). In the case of growth factors, recent studies have implicated a positively charged, 7 amino-acid stretch in acidic fibroblast growth factor (aFGF) as responsible for targeting to the nucleus and required for mitogenic activity as well (Imamura et al. 1990). There are reports that PDGF can achieve a nuclear location (Lee et al. 1987; Yeh et al. 1987), and there is evidence that the nuclear targeting signal (Lee et al. 1987) corresponds to the region identified by us as a membrane retention domain. Our present studies demonstrate that deletion of this putative nuclear targeting sequence from either chain of PDGF had no effect on its mitogenic properties. Therefore, if such a basic amino acid stretch were also functional in PDGF nuclear targeting, it does not appear to be required for the proliferative response to this growth factor.

In addition to PDGF, the prototype of another family of structurally related growth factors, int-1, has been shown to remain tightly associated with the producing cell (Papkoff and Schryver 1990). The carboxy-terminal region of the int-1 protein contains a similarly charged series of amino acids conserved in both mouse and human homologs (van Ooyen and Nusse 1984; van Ooyen et al. 1985). Thus, the mechanism described here for the cell-surface retention of PDGF-A and PDGF-B may be applicable to other growth factors as well.

\section{Materials and methods}

Construction of PDGF recombinants

Chimeric and stop mutant PDGF molecules were engineered by 
the site-directed mutagenesis method of Kunkel, through which individual codons of PDGF-A or PDGF-B were altered (Zoller and Smith 1982; Kunkel 1985). Mutants were identified, and their DNA sequence was verified by the dideoxynucleotide sequencing method (Sanger et al. 1977). Chimeras recombined PDGF-A and PDGF-B cDNAs through common restriction endonuclease sites. Briefly, the PDGF- $\mathrm{A}^{187} \mathrm{~B}^{190}$ and PDGF$\mathrm{B}^{188} \mathrm{~A}^{187}$ chimeras were joined at a preexisting MroI site in PDGF-A and an Mrol site introduced into PDGF-B. The nomenclature for chimeras indicates the PDGF-A and PDGF-B codons at which the recombination was performed. Stop mutants were made by introduction of a TAA or TAG stop codon at the indicated codon and verified by DNA sequence analysis. Chimeras or stop mutants were then transferred into MMTneo for transfection.

\section{Transfection analysis}

Plasmid DNA from each recombinant was introduced into NIH-3T3 cells by transfection with the recombinant plasmid DNA and $40 \mu \mathrm{g}$ of carrier calf thymus DNA by the calcium phosphate precipitation technique (Wigler et al. 1977). Transfected cultures were scored for colony formation in the presence of G418 or for focus-forming activity 2-3 weeks after transfection. Colony formation following selection in medium containing geneticin was utilized as an internal marker of transfection efficiency (LaRochelle et al. 1990).

\section{Immunoprecipitation}

After geneticin selection, NIH-3T3 cells were metabolically labeled for $4 \mathrm{hr}$ with $\left[{ }^{35} \mathrm{~S} /\right.$ methionine $(125 \mu \mathrm{Ci} / \mathrm{ml})$ and $\left[{ }^{35} \mathrm{~S}\right]$ cysteine $(125 \mu \mathrm{Ci} / \mathrm{ml})$ (1200 Ci/ mmole; Amersham) in methionine- and cysteine-free Dulbecco's modified Eagle minimal essential mediurn (DMEM) containing $25 \mu \mathrm{M}$ zinc chloride (LaRochelle et al. 1990). Conditioned medium and crude membrane fractions were prepared as described (Robbins et al. 1985). Peptide antisera against amino (residues 23-37)- and carboxy (residues 227-241)-terminal domains of PDGF-B protein have been described (Robbins et al. 1983; Igarashi et al. 1987). Analogous antisera against the amino (residues $72-87$ )- and carboxy (residues 185-199)-terminal regions of the PDGF-A, as well as a neutralizing PDGF antibody, have also been reported (Robbins et al. 1982; Igarashi et al. 1987; LaRochelle et al. 1990). We also developed a PDGF-A antiserum to carboxy-terminal residues 200-211 for use in the present studies. For immunoprecipitations, conditioned media or crude membranes were incubated with primary antisera for $1 \mathrm{hr}$ at $4^{\circ} \mathrm{C}$. Immunoprecipitates were recovered with Staphylococcus aureus protein A-Sepharose (Pharmacia). After solubilization by boiling in SDS-PAGE sample buffer, proteins were analyzed by SDS-PAGE on $14 \%$ polyacrylamide gels.

\section{Mitogenic assay}

Mitogenic assays were performed as described previously. Briefly, NIH-3T3 cells were seeded at $2 \times 10^{4}$ cells/well into 96-well plates in DMEM-10\% calf serum. Cells were allowed to become quiescent over $4-6$ days at $37^{\circ} \mathrm{C}$. Plasmid-transfected NIH-3T3 cells were fractionated into crude membrane fractions or conditioned medium as described (Robbins et al. 1985). For mitogenic assay, the preparations were added to quiescent $\mathrm{NIH}$ $3 \mathrm{~T} 3$ cultures. After $16 \mathrm{hr},\left[{ }^{3} \mathrm{H}\right]$ thymidine $(2 \mu \mathrm{Ci} /$ well $)$ was also added to the cells for an additional 5 -hr period and DNA was harvested. $\left[{ }^{3} \mathrm{H}\right]$ Thymidine incorporation was measured by liquid scintillation counting.

\section{Membrane extraction of PDGF}

Crude membrane fractions subjected to various extraction protocols were analyzed for the presence or absence of PDGF. Briefly, the indicated treatment was performed for 30 min with radiolabeled crude membrane fractions of transfectants initially suspended in $10 \mathrm{~mm}$ potassium phosphate buffer $(\mathrm{pH} 7.4)$. The crude membranes were fractionated from the supernatant by centrifugation, and each fraction was assayed for radiolabeled PDGF by immunoprecipitation with anti-PDGF sera as described.

\section{Antibody surface absorption and suramin release of PDGF}

NIH-3T3 transfectants were radiolabeled as described and then treated with a PDGF polyclonal antibody for $30 \mathrm{~min}$ at $4^{\circ} \mathrm{C}$. Unbound antibody was removed by washing. The cells were lysed, and remaining antibody bound to cell surface PDGF was immunoprecipitated with protein A-Sepharose. For suramin treatment, cells were radiolabeled in the presence or absence of $100 \mu \mathrm{M}$ suramin for $3.5 \mathrm{hr}$ and the conditioned media were subjected to immunoprecipitation with the appropriate antibody followed by protein A-Sepharose. In some cases, PDGF-A transfectants were treated with $10 \mu \mathrm{M}$ monensin overnight and radiolabeled for $3.5 \mathrm{hr}$ in the presence of $10 \mu \mathrm{M}$ monensin. Crude cell membranes were then treated with $100 \mu \mathrm{M}$ suramin, and the released PDGF-A was immunoprecipitated. SDS-PAGE was performed as described above.

\section{Acknowledgments}

We thank Marthanna Moore and James McArdle for their expert technical assistance.

The publication costs of this article were defrayed in part by payment of page charges. This article must therefore be hereby marked "advertisement" in accordance with 18 USC section 1734 solely to indicate this fact.

\section{References}

Beckman, M.P., C. Betsholtz, C.-H. Heldin, B. Westermark, E. Di Marco, P.P. Di Fiore, K.C. Robbins, and S.A. Aaronson. 1988. Comparison of the biological properties and transforming potential of human PDGF-A and PDGF-B chains. Science 241: 1346-1349.

Devare, S.G., E.P. Reddy, J.D. Law, K.C. Robbins, and S.A. Aaronson. 1983. Nucleotide sequence of the simian sarcoma virus genome: Demonstration that its acquired cellular sequences encode the transforming gene product, $\mathrm{p} 28^{\text {sis }}$. Proc. Natl. Acad. Sci. 80: 731-735.

Doolittle, R F., M.W. Hunkapiller, L.E. Hood, S.G. Devare, K.C. Robbins, S.A. Aaronson, and H.N. Antoniades. 1983. Simian sarcoma virus onc gene, $\mathrm{v}$-sis, is derived from the gene or genes encoding a platelet-derived growth factor. Science 221: 275-277.

Flanagan, J.G. and P. Leder. 1990. The kit ligand: A cell surface molecule altered in steel mutant fibroblasts. Cell 63: 185194.

Flanagan, J.G., D.C. Chan, and P. Leder. 1991. Transmembrane form of the kit ligand growth factor is determined by alternative splicing and is missing in the $\mathrm{Sl}^{\mathrm{d}}$ mutant. Cell 64: 1025-1035.

Gray, A., T.J. Dull, and A. Ullrich. 1983. Nucleotide sequence of epidermal growth factor cDNA predicts a 128,000 molecular weight protein precursor. Nature 303: 722-725.

Huang, E., K. Nocka, D.R. Beire, T.-Y. Chu, J. Buck, H.-W. 
Lahm, D. Wellner, P. Leder, and P. Besmer. 1990. The hematopoietic growth factor $\mathrm{KL}$ is encoded by the $S I$ locus and is the ligand of the c-kit receptor, the gene product of the $W$ locus. Cell 63: 225-233.

Igarashi, H., C.D. Rao, M. Siroff, F. Leal, K.C. Robbins, and S.A. Aaronson. 1987. Detection of PDGF-2 homodimers in human tumor cells. Oncogene 1: 79-85.

Imamura, T., K Engleka, X. Zhan, Y. Tokita, R. Forough, D. Roeder, A. Jackson, J.A.M. Maier, T. Hla, and T. Maciag. 1990. Recovery of mitogenic activity of a growth factor mutant with a nuclear translocation sequence. Science 249: $1567-1570$.

Jackson, M.R., T. Nilsson, and P.A. Peterson. 1990. Identification of a consensus motif for retention of transmembrane proteins in the endoplasmic reticulum. $E M B O / .9: 3153-$ 3162 .

Kalderon, D., B.L. Roberts, W.D. Richardson, and A.E. Smith. 1984. A short amino acid sequence able to specify nuclear location. Cell 39: 499-509.

King, C.R., N.A. Giese, K.C. Robbins, and S.A. Aaronson. 1985. In vitro mutagenesis of the $\mathrm{v}$-sis transforming gene defines functional domains of its growth factor-related product. Proc. Natl. Acad. Sci. 82: 5295-5299.

Kunkel, T.A. 1985. Rapid and efficient site-specific mutagenesis without phenotypic selection. Proc. Natl. Acad. Sci. 82: 488-492.

LaRochelle, W.J., N. Giese, M. May-Siroff, K.C. Robbins, and S.A. Aaronson. 1990. Molecular localization of the transforming and secretory properties of PDGF A and PDGF B. Science 248: 1541-1544.

Lee, B.A., D.W. Maher, M. Hannink, and D.J. Donoghue. 1987. Identification of a signal for nuclear targeting in plateletderived growth factor-related molecules. Mol. Cell. Biol. 7: 3527-3537.

Massague, J. 1990. Transforming growth factor- $\alpha$. J. Biol. Chem. 265: 21393-21396.

Munro, S. and H.R.B. Pelham. 1987. A C-terminal signal prevents secretion of luminal ER proteins. Cell 48: 899-907.

Nilsson, T., M.R. Jackson, and P.A. Peterson. 1989. Short cytoplasmic sequences serve as retention signals for transmembrane proteins in the endoplasmic reticulum. Cell 58: 707718.

Ostman, A., L. Rall, A. Hammacher, M.A. Wormstead, D. Coit, P. Valenzuela, C. Betsholtz, B. Westermark, and C.-H. Heldin. 1988. Synthesis and assembly of a functionally active recombinant platelet-derived growth factor $A B$ heterodimer. I. Biol. Chem. 263: 16202-16208.

Papkoff, J. and B. Schryver. 1990. Secreted int-1 protein is associated with the cell surface. Mol. Cell. Biol. 10: 2723-2730.

Rettenmier, C.W. and M.F. Roussel. 1988. Differential processing of colony-stimulating factor-1 precursors encoded by two human cDNAs. Mol. Cell. Biol. 8: 5026-5031.

Robbins, K.C., S.G. Devare, E.P. Reddy, and S.A. Aaronson. 1982. In vivo identification of the transforming gene product of simian sarcoma virus. Science 218: 1131-1133.

Robbins, K.C., H.N. Antoniades, S.G. Devare, M.W. Hunkapiller, and S.A. Aaronson. 1983. Structural similarities between sarcoma virus gene product(s) and human plateletderived growth factor. Nature 305: 605-608.

Robbins, K.C., F. Leal, J.H. Pierce, and S.A. Aaronson. 1985. The v-sis/PDGF-2 transforming gene product localizes to cell membranes but is not a secretory protein. EMBO I. 4: 17831792.

Rorsman, F., M. Bywater, T.J. Knott, J. Scott, and C. Betsholtz. 1988. Structural characterization of the human platelet-derived growth factor A-chain cDNA and gene: Alternative exon usage predicts two different precursor proteins. Mol. Cell. Biol. 8: 571-577.

Sanger, F., S. Nicklen, and A.R. Carlson. 1977. DNA sequencing with chain-terminating inhibitors. Proc. Natl. Acad. Sci. 74: 5463-5467.

Scott, I., M. Urdea, M. Quiroga, R. Sanchez-Pescador, N. Fong, M. Selby, W. J. Rutter, and G.I. Bell. 1983. Structure of a mouse submaxillary messenger RNA encoding epidermal growth factor and seven related proteins. Science 221: 236240.

van Ooyen, A. and R. Nusse. 1984. Structure and nucleotide sequence of the putative mammary oncogene int- 1 ; proviral insertions leave the protein-encoding domain intact. Cell 39: 233-240.

van Ooyen, A., V. Kwee, and R. Nusse. 1985. The nucleotide sequence of the human int-1 mammary oncogene; evolutionary conservation of coding and non-coding sequences. EMBO J. 4: 2905-2909.

Waterfield, M.D., G.T. Scrace, N. Whittle, P. Stroobant, A. Johnsson, A. Wasteson, B. Westermark, C.-H. Heldin, H.S. Huang, and T.F. Deuel. 1983. Platelet-derived growth factor is structurally related to the putative transforming protein p28 $8^{\text {sis }}$ of simian sarcoma virus. Nature 304: 35-39.

Wigler, M., S. Silverstein, L.-S. Lee, A. Pellicer, Y.-C. Cheng, and R. Axel. 1977. Transfer of purified herpes virus thymidine kinase gene to cultured mouse cells. Cell 11: 223-232.

Yeh, H.-I., G.F. Pierce, and T.F. Deuel. 1987. Ultrastructural localization of a platelet-derived growth factor/v-sis-related protein(s) in cytoplasm and nucleus of simian sarcoma virus transformed cells. Proc. Natl. Acad. Sci. 84: 2317-2321.

Zoller, M.J. and M. Smith. 1982. Oligonucleotide-directed mutagenesis using M13-derived vectors: An efficient and general procedure for the production of point mutations in any fragment of DNA. Nucleic Acids Res. 10: 6487-6501. 


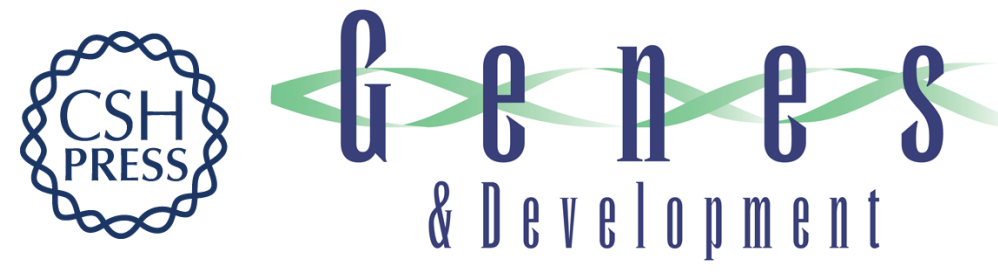

\section{A novel mechanism regulating growth factor association with the cell surface: identification of a PDGF retention domain.}

W J LaRochelle, M May-Siroff, K C Robbins, et al.

Genes Dev. 1991, 5:

Access the most recent version at doi:10.1101/gad.5.7.1191

References This article cites 33 articles, 17 of which can be accessed free at: http://genesdev.cshlp.org/content/5/7/1191.full.html\#ref-list-1

License

Email Alerting

Service

Receive free email alerts when new articles cite this article - sign up in the box at the top right corner of the article or click here.

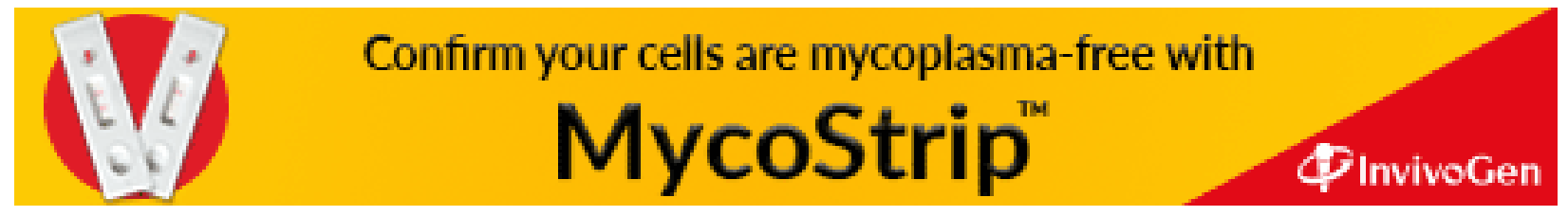

